\title{
Can One-Minute Preceptor and SNAPPS Improve Your Inpatient Teaching?
}

\author{
Jessica Zeidman, MD, Meridale Baggett, MD, Daniel P. Hunt, MD*
}

Division of General Internal Medicine, Department of Medicine, Massachusetts General Hospital, Boston, Massachusetts.

Hospitalists who teach in the clinical environment face challenges that include increased workload, ${ }^{1}$ perception among trainees that there is less time to teach, ${ }^{2}$ and competition with electronic devices for teaching engagement. ${ }^{3,4}$ In view of these and other challenges, we believe there is potentially much to gain from considering and adapting educational techniques that have been successful in nonhospital and even nonmedical domains. Innovative teaching methods include those designed for the grade-school classroom (Courage to Teach, ${ }^{5}$ Teaching With Love and $\operatorname{Logic}^{6}$ ), and the business world (Teaching Smart People How to Learn, ${ }^{7}$ The Back of the Napkin ${ }^{8}$ ), among other nonmedical professions. Within medicine, we can also re-examine strategies long utilized in the ambulatory setting. Pascoe and colleagues offer an important example of this in their review of oneminute preceptor (OMP) and SNAPPS, techniques developed by our colleagues in the outpatient setting but with great potential for framing discussion of clinical reasoning in the inpatient space. ${ }^{9}$

Applying OMP and SNAPPS to inpatient teaching presents some challenges but also genuine opportunities not found in traditional outpatient teaching. As noted by the authors, unlike the solitary learner typical of the outpatient setting, in the inpatient setting the attending is more commonly working with a group of learners of multiple levels and sometimes multiple disciplines. Furthermore, the supervising resident typical of inpatient teams is a learner who inhabits the roles of both trainee and teacher. One can imagine that if OMP and SNAPPs are applied with absolute fidelity to the inpatient setting, without reflection on venue, the teaching encounter might be overly focused on the presenting learner, leaving the rest of the team unattended to, disengaged, and not benefitting from the models. Therefore, attention to group engagement in the process is necessary for successful adaptation. Both models have the potential to

\footnotetext{
*Address for correspondence and reprint requests: Daniel P. Hunt, MD, Director, Inpatient Clinician Educator Service, Department of Medicine, Massachusetts General Hospital, 50 Staniford Street, Suite 503B, Boston, MA 02114; Telephone: 617-643-0581; Fax: 617-724-9428; E-mail: dphunt@partners.org
}

Received: November 24, 2014; Accepted: November 26, 2014 2015 Society of Hospital Medicine DOI 10.1002/jhm.2307 Published online in Wiley Online Library (Wileyonlinelibrary.com). help organize the group dynamic during rounds to promote broad participation. The authors describe some examples of how to engage various group members in different steps. It is worth highlighting a few key themes that enable successful use of these models in the inpatient setting.

One key theme is to teach the model to the supervising resident at the beginning of the rotation and agree, before rounds, how the attending and resident will interact as coleaders of the discussion. Because these models offer a stepwise approach to going through a case with a learner, they have the potential to demystify the teaching process, offering an accessible framework for supervising residents to learn teaching both by practicing and by comprehending what their attending is doing to lead a team through a case discussion. With attending support, the supervising resident can be encouraged to manage the team discussion, leading the team using either approach. It can be helpful to touch base briefly before rounds each day to define the teaching roles, giving the resident progressively more responsibility leading the discussion as the rotation progresses.

Another key theme is to use graduated participation. As the authors note, the group must be engaged in the discussion, and the example scenarios illustrate each step of the models being applied to the group. To ensure that the entire group remains eager to partake, the leader must maintain a nonthreatening teaching atmosphere, organizing participation in a way that does not shame learners or undermine the roles people inhabit. To this end, it can be helpful to direct questions to particular members or levels of the group at a time. When expanding participation around a specific question or concept, always work from junior members to senior members, never imposing the reverse. This principle is clearly not exclusive to using these models, but is requisite to successful adaptation of these traditionally dyadic models, in which there is no particular attention to group dynamics within the framework.

A third key theme is to utilize the unique expertise of the other health professionals on the team in steps 4, 5, and 6 of SNAPPS and step 3 of OMP. In step 4 and 5 of SNAPPS, when the teaching attending introduces the team to the model, it is important to encourage them to probe not just the teacher but other disciplines on the team for input. In the inpatient setting, these steps provide an organized point in 
the discussion in which to involve the other members of the professional team, modeling collaborative interdisciplinary practice.

As Pascoe et al. point out, there are limited studies of OMP and SNAPPS as teaching models in the inpatient environment. This should stimulate academic hospitalists with interest in medical education research to consider how these models might be studied. For example, in comparison to traditional inpatient teaching rounds, do these approaches provide equivalent content coverage? How do they impact the efficiency of teaching rounds? Are attendings who consistently apply these models more effective in providing feedback or assessing training milestones? How much training and practice is required to incorporate these teaching models in the inpatient environment?

Given the time pressure and increasing complexity of medical care in the hospital, coupled with the evolving needs and resources of our learners, we must seek innovative educational practices from sources outside our hospitals to provide the best possible training in hospital medicine. An outstanding recent review by Martin et al. provided an overview of other strategies for teaching in today's environment. ${ }^{10} \mathrm{We}$ also have much to learn from our colleagues in outpa- tient medicine, not only in clinical care, but also in medical education. And we have much that we have learned about teaching as hospitalists that needs to be more broadly disseminated.

Disclosure: Nothing to report.

\section{References}

1. Desai SV, Feldman L, Brown L, et al. Effect of the 2011 vs 2003 duty hour regulation-compliant models on sleep duration, trainee education, and continuity of patient care among internal medicine house staff: a randomized trial. JAMA Intern Med. 2013;173(8):649-655.

2. Mazotti LA, Vidyarthi AR, Wachter RM, Auerbach AD, Katz PP. Impact of duty-hour restriction on resident inpatient teaching. J Hosp Med. 2009;4(8):476-480.

3. Verghese A. Culture shock-patient as icon, icon as patient. N Engl J Med. 2008;359(26):2748-2751.

4. Katz-Sidlow RJ, Ludwig A, Miller S, Sidlow R. Smartphone use during inpatient attending rounds: prevalence, patterns and potential for distraction. J Hosp Med. 2012;7(8):595-599.

5. Palmer PJ. The Courage to Teach: Exploring the Inner Landscape of a Teacher's Life. San Francisco, CA: Jossey-Bass; 2007.

6. Fay J, Funk D. Teaching With Love \& Logic: Taking Control of the Classroom. 1st ed. Golden, CO: Love and Logic Press; 1995.

7. Argyris C. Teaching Smart People How to Learn. Boston, MA: Harvard Business Press; 2008.

8. Roam D. The Back of the Napkin: Solving Problems and Selling Ideas With Pictures. New York, NY: Portfolio; 2008.

9. Pascoe JM, Nixon J, Lang VJ. Maximizing teaching on the wards: review and application of the one-minute preceptor and SNAPPS models. J Hosp Med. 2015;10(2):125-130.

10. Martin SK, Farnan JM, Arora VM. Future: new strategies for hospitalists to overcome challenges in teaching on today's wards. J Hosp Med. 2013;8(7):409-413. 